\title{
Acute diarrhoea in a community cohort of children who received an oral rotavirus vaccine in Northeast Brazil
}

\author{
Sarah Cristina Fontes Vieira', Ricardo Queiroz Gurgel ${ }^{1 /+}$, Andrew Kirby ${ }^{2}$, Isis Pinheiro Barreto ${ }^{3}$, \\ Liane Desiderio de Souza ${ }^{3}$, Oderlan Carvalho Oliveira ${ }^{3}$, Jailson de Barros Correia ${ }^{4}$, \\ Winifred Dove ${ }^{2}$, Nigel A Cunliffe ${ }^{2}$, Luis E Cuevas ${ }^{5}$ \\ ${ }^{1}$ Núcleo de Pós-Graduação em Medicina ${ }^{3}$ Escola de Medicina, Universidade Federal de Sergipe, Aracaju, SE, Brasil \\ ${ }^{2}$ Institute of Infection and Global Health, Clinical Infection, Microbiology and Immunology ${ }^{5}$ Liverpool School of Tropical Medicine, \\ University of Liverpool, Liverpool, UK ${ }^{4}$ Instituto de Medicina Integral Professor Fernando Figueira, Recife, PE, Brasil
}

Rotavirus is an important cause of childhood diarrhoea. A monovalent rotavirus vaccine (Rotarix ${ }^{\circledR}$ ) was introduced into the Immunization Program of Brazil in 2006. In this study, we describe the incidence and burden of disease of rotavirus diarrhoea in two cohorts of children (vaccinated and unvaccinated). We followed two groups of 250 children under one year old, who were enrolled in December 2006 from a low-income residential area in Northeast Brazil. The children were monitored every two weeks for two years. Stool samples from children with diarrhoea were examined for the presence of rotavirus. Rotaviruses were genotyped using real time-polymerase chain reaction. The mean numbers of all-cause diarrhoea episodes/child (adjusted for age) in the first year were 0.87 and 0.84 , in vaccinated and unvaccinated children, respectively. During the second year, the number of episodes/ child decreased to 0.52 and 0.42 . Only $16(4.9 \%)$ of 330 stool samples were rotavirus-positive (10 vaccinated and 6 unvaccinated children) and only P [4]G2 rotaviruses were identified. All-cause diarrhoea episodes were more severe in unvaccinated children in the first year of age $(p<0.05)$, while vaccinated children had more severe episodes 18 months after vaccination. Rotavirus diarrhoea incidence was very low in both groups.

Key words: diarrhoea - rotavirus - vaccine - cohort - children - Brazil

Acute diarrhoea is one of the main causes of childhood morbidity and mortality worldwide (Bishop 2009). Although major improvements in sanitation and the availability of oral rehydration therapy have reduced childhood diarrhoea mortality in recent decades, Brazil included, these reductions vary from region to region (Sergio \& Leon 2009). Rotavirus is the main cause of severe diarrhoea, causing 600,000 deaths and two million hospitalizations annually worldwide (Parashar et al. 2006). Almost all children experience at least one episode of rotavirus gastroenteritis before reaching five years of age (Valencia-Mendoza et al. 2008) and the main strategy to control rotavirus diarrhoea is the widespread use of vaccines (Bishop 2009).

Brazil was one of the pioneer countries; an oral rotavirus vaccine was introduced into its National Immunization Program (EPI) in March 2006. This is in agreement with current World Health Organization guidelines, which recommend the inclusion of rotavirus vaccines in the EPI of countries where the vaccine effectiveness suggests a significant impact on public health (WHO 2007). A study undertaken in the state of Sergipe (SE), located in

Financial support: $\mathrm{CNPq}$

NAC was the principal investigator overseeing a clinical trial of Rotarix $^{\circledR}$ in Malawi. All other authors have no conflict of interest to declare. + Corresponding author: ricardoqg@infonet.com.br

Received 24 October 2010

Accepted 23 February 2011
Northeast Brazil, demonstrated that rotavirus vaccination resulted in a significant decrease in the number of rotavirus-related hospitalizations and consultations (Centenari et al. 2010); however, it is understood that hospital-based studies often report a higher incidence of rotavirus than community-based studies (Patel et al. 2007, Sáfadi et al. 2010). This over-representation is due to the fact that rotavirus causes more severe disease than other pathogens. Because of this, vaccine effectiveness is likely to be higher in these settings, since rotavirus vaccines consistently demonstrate higher efficacy against more severe rotavirus disease outcomes (Soares-Weiser et al. 2010).

No studies have examined the effectiveness of the rotavirus vaccine on rotavirus infections that are community-based. In this study, we describe the incidence of all-cause and rotavirus-derived diarrhoea in a cohort of children residing in a community-based setting in Northeast Brazil. These children were vaccinated with an oral monovalent rotavirus vaccine and the burden of disease was compared to that of an unvaccinated cohort of children residing in the same community.

\section{PATIENTS, MATERIALS AND METHODS}

This was a longitudinal cohort study of children under two years of age residing in the district of Santa Maria, on the outskirts of the city of Aracaju (SE). Santa Maria is a low-income housing state located near the main metropolitan area; the two are linked with a paved road and common public services. Three government Health Centres located within the community serve the area. Children greater than or equal to four months of age registered in Santa Maria's Health Centre vaccination facili- 
ties were visited at home in 2006 and their parents were invited to enrol their children in the study, independent of their vaccination status. The parents of the first 250 children who had received two oral doses of the Rotarix ${ }^{\circledR}$ vaccine (GlaxoSmithKline Biologicals, Rixensart, Belgium) at two and four months of age (vaccinated cohort) were invited to participate and a further 250 children who had not received the vaccine comprised the unvaccinated cohort. Vaccination status was verified using the child's vaccination card and the Health Centre's databases. The youngest unvaccinated children registered were selected, despite the fact that the two groups could not be matched by age, as unvaccinated children had to be ineligible for vaccination at the time of enrolment, and therefore at least four months of age. Unvaccinated children serving as the control group were not offered the vaccine due to EPI guidelines that exclude children greater than or equal to four months of age to avoid the risk of intussusception among older infants (WHO 2007).

After obtaining informed parental consent, all children were visited at home for the following two years, independently of their age at the time of enrolment. Visits were conducted every two weeks by three trained interviewers who inquired as to whether or not the child had experienced diarrhoea episodes during the previous twoweek period. If the parent indicated that the child had diarrhoea, the interviewer collected information regarding the date of the episode and clinical presentation. The severity of the diarrhoea episodes was classified following Ruuska and Vesikari score (Ruuska \& Vesikari 1990), as modified by Nakagomi et al. (2005). Episodes with scores equal to or above 12 were classified as severe and analysed according to age categories to control for the age differences in vaccinated and unvaccinated children.

Stool samples were collected for all diarrhoea episodes encountered during home visits. Acute diarrhoea was defined as any episode with more than three watery stools per day and duration of less than 14 days. Parents were asked to collect the stool samples at home and to bring the samples to the Health Centres the next day or to phone the research staff, which would collect the samples at home if the parent was unable to attend the Health Centre. Some parents missed collecting samples at the time of the episodes, which resulted in more diarrhoea episodes being reported than the number of stool samples collected. Stools collected were stored in a refrigerator for a maximum of $24 \mathrm{~h}$ and were subsequently stored at $-80^{\circ} \mathrm{C}$ until analysis. Rotavirus antigen was detected by using an enzyme-linked immunosorbent assay (ELISA) (Rotaclone, Meridian Bioscience, Inc, Cincinnati, OH, USA). Genomic RNA was extracted from positive samples using previously described protocols (Gentsch et al. 1992) and the type of rotavirus ( $\mathrm{G}$ or $\mathrm{P}$ ) was determined using hemi-nested multiplex real time-polymerase chain reaction with consensus and type-specific primers, as described previously (Gouvea et al. 1990, Gentsch et al. 1992).

The Ethics Research Committee of the Sergipe Federal University Hospital and the Research Ethics Committee of the Liverpool School of Tropical Medicine approved the protocols utilised in 2006 (CAAE 0839.0.000.107-06 and CAAE 009.0.107.000-07).

\section{RESULTS}

Fifty-six (11.2\%) of the 500 participants enrolled were lost during follow-up resulting in 222 vaccinated and 222 unvaccinated children being included in the final analysis. Children in the two cohorts had similar housing conditions and access to pipe water and drains. The mean (SD) age of vaccinated and unvaccinated children was 6.6 (3.1) months and 15.3 (4.9) months at the time of enrolment, respectively $(p<0.001)$ (Table I). The older age of the latter was due to the rapid uptake of the vaccine soon after vaccine introduction, with very few young children not being vaccinated at the time of recruitment.

The cumulative incidence of diarrhoea was low in both cohorts, with 87 and 84 episodes per 100 children reported in the vaccinated and unvaccinated cohorts during the first year of follow-up and 52 and 42 episodes per 100 children in the second year, respectively $(p<$ 0.05 for the difference between first and second year of both cohorts). Diarrhoea incidence was stratified by age to control for the differences in age, as shown in Fig. 1. The peak incidence was observed during the second six-month period of the study in three-12-month-old vaccinated children and in seven-12-month-old unvac-

\section{TABLE I}

Characteristics of study participants

\begin{tabular}{|c|c|c|c|}
\hline & $\begin{array}{c}\text { Vaccinated } \\
(\mathrm{n}=222) \\
\mathrm{n}(\%)\end{array}$ & $\begin{array}{l}\text { Unvaccinated } \\
\qquad \begin{array}{c}\mathrm{n}=222) \\
\mathrm{n}(\%)\end{array}\end{array}$ & $\mathrm{p}$ \\
\hline Age (months) [mean (SD)] & $6.6(3.1)$ & $15.3(4.9)$ & $<0.001$ \\
\hline Male & $113(50.9)$ & $109(49.1)$ & 0.7 \\
\hline Rooms at home [mean (SD)] & $5(1.6)$ & $4.9(3.6)$ & 0.81 \\
\hline \multicolumn{4}{|l|}{ Sewerage } \\
\hline Piped & $120(54.5)$ & $98(45.4)$ & 0.12 \\
\hline Well & $96(43.6)$ & $115(53.2)$ & \\
\hline None & $4(1.8)$ & $3(1.4)$ & \\
\hline \multicolumn{4}{|l|}{ Toilet } \\
\hline With flusher & $158(73.8)$ & $165(76.4)$ & 0.51 \\
\hline Without flusher & $49(22.9)$ & $42(19.4)$ & \\
\hline Latrine & $3(1.4)$ & $1(0.5)$ & \\
\hline None & $4(1.9)$ & $8(3.7)$ & \\
\hline Water source (Public) & $214(98.6)$ & $213(99.1)$ & 0.76 \\
\hline \multicolumn{4}{|l|}{ Piped water } \\
\hline Inside & $215(96.8)$ & $213(96.4)$ & 0.94 \\
\hline Outside & $4(1.8)$ & $5(2.3)$ & \\
\hline None & $3(1.4)$ & $3(1.3)$ & \\
\hline \multicolumn{4}{|l|}{ House type } \\
\hline Brick & $215(97.7)$ & $215(97.7)$ & 1.0 \\
\hline Other & $5(2.3)$ & $5(2.3)$ & \\
\hline \multicolumn{4}{|l|}{ Open sewerage } \\
\hline In front of house & $102(46.1)$ & $101(46.1)$ & 0.91 \\
\hline Far from house & $41(18.6)$ & $44(20.1)$ & \\
\hline None & $78(35.3)$ & $74(33.8)$ & \\
\hline
\end{tabular}


cinated children. The incidence of diarrhoea was higher during the rainy season, but the overall number of cases decreased in the second year (Fig. 2).

A total of 581 episodes were documented to calculate the severity score. Of these, 273 (47\%) occurred in vaccinated and 308 (53\%) in unvaccinated children. The mean severity score of all-cause diarrhoea stratified by age group is shown in Table II. Children who had been recently vaccinated (7-12 months-old) had lower mean severity scores than the unvaccinated controls [means (SD) of 5.6 (2.8) and 8.9 (4.2), respectively, p < 0.046]. This difference disappeared in children aged 13-24 months, with vaccinated children having similar allcause severity scores to the unvaccinated controls. The difference was reversed in children older than 24 months with vaccinated children having more severe episodes than the unvaccinated controls [means (SD) 7.9 (4.7) and 6.2 (4.2), respectively, $\mathrm{p}=0.02]$.

A total of 327 stool samples were collected and tested for rotavirus (180 from vaccinated and 147 from unvaccinated children). Sixteen $(4.8 \%)$ samples were rotavirus-ELISA positive, 10 of which (5.5\%) occurred in vaccinated and six (4\%) occurred in unvaccinated children $(\mathrm{p}>0.5)$. The proportion of severe episodes (score $\geq$ 12) was analysed by comparing rotavirus-positive and rotavirus-negative children based on their vaccination status. Among vaccinated children, the proportion of diarrhoea episodes with a score $>12$ was similar in rotavi-
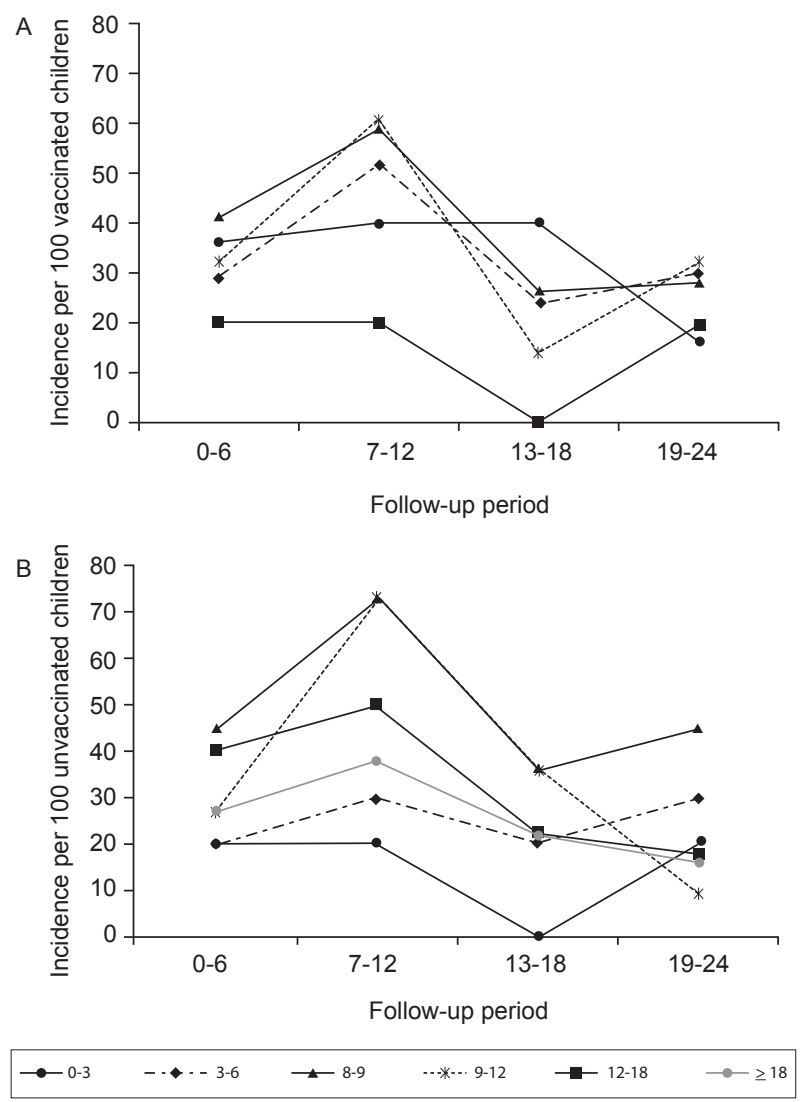

Fig. 1: incidence of acute diarrhoea in vaccinated (A) and unvaccinated (B) children during the 24-month follow-up period by age. rus-positive and rotavirus-negative cases [5 (59\%) of the 10 rotavirus-positive and $12(27.1 \%)$ of the 170 rotavirusnegative, respectively, $p=0.15$ ]. However, among unvaccinated children, the proportion of diarrhoea episodes with a score $\geq 12$ was higher in the rotavirus-positive cases than in rotavirus-negative cases $[(4,67 \%)$ of 6 rotavirus-positive and $23(16.3 \%)$ of 11 rotavirus-negative, respectively, Fisher exact test $(p=0.001)]$.

The rotavirus $\mathrm{P}[4] \mathrm{G} 2$ genotype was identified in seven of the 10 vaccinated children and three of the six unvaccinated children. The remaining strains were rotavirus non-typeable (NT); with three P[NT]GNT rotavirus strains recovered from vaccinated children and two from unvaccinated children. One P[NT]G2 sample was identified in an unvaccinated child.

\section{DISCUSSION}

This is the first description of a cohort of children vaccinated with an oral rotavirus vaccine, followed up in a low-income community setting, in the context of routine childhood immunization in Brazil. Surprisingly, the overall incidence of all-cause diarrhoea was lower than expected and was similar between vaccinated and unvaccinated children, after stratifying by age. Additionally, the incidence decreased with time in all age groups and the total number of episodes decreased from 20072008. According to Aracaju's Public Health Department (www.datasus.gov.br), acute diarrhoea incidence in the

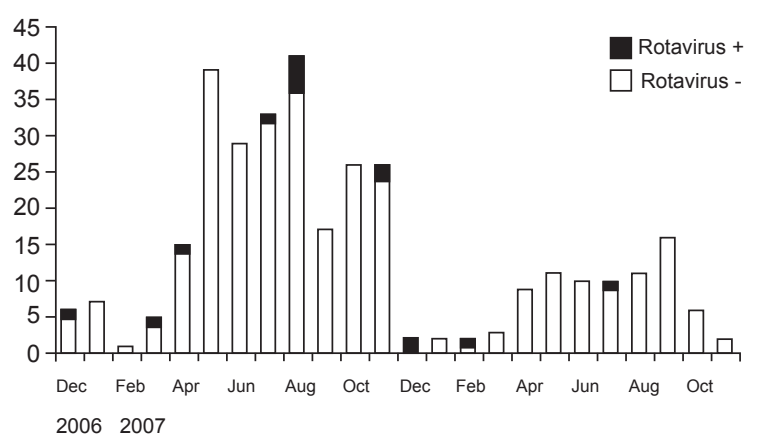

Fig. 2: number of stool samples collected and proportion of samples with rotavirus in vaccinated and unvaccinated children, Santa Maria, Aracaju, Sergipe, Brazil, December 2006-November 2008.

\section{TABLE II}

Mean severity score of all-cause diarrhoea by vaccination status and age group

\begin{tabular}{lccccc}
\hline Age (months) & Vaccinated & & Unvaccinated & $\mathrm{p}$ \\
\hline & $\mathrm{n}$ & Mean (SD) & \multicolumn{3}{c}{ Mean (SD) } \\
& score & $\mathrm{n}$ & score \\
$0-6$ & 1 & $3(0)$ & 6 & $6.5(4.1)$ & 0.5 \\
$7-12$ & 7 & $5.6(2.8)$ & 70 & $8.9(4.2)$ & 0.05 \\
$13-18$ & 44 & $8.8(4.2)$ & 109 & $8.9(4.6)$ & 0.18 \\
$19-24$ & 90 & $8.1(4.3)$ & 69 & $8.9(4.8)$ & 0.2 \\
$>24$ & 129 & $7.9(4.7)$ & 54 & $6.2(6.2)$ & 0.02 \\
\hline
\end{tabular}


study area decreased from 59.8-52.5 episodes per 1,000 children during the two years this study was conducted and the higher incidence observed among the cohorts is likely reflects the increased sensitivity of active household surveillance, which identified mild cases that were managed at home, as previously described (Banerjee et al. 2006, Vernacchio et al. 2006).

In addition to the low incidence of all-cause diarrhoea, very few children had documented rotavirusborne diarrhoea during the two years this study was conducted. This observation is in agreement with the lower incidence of rotavirus infections reported in SE after the vaccine was introduced (Gurgel et al. 2009) and may also reflect the location of the study, as the proportion of rotavirus cases in community-based studies is characteristically lower than in hospital-based studies (Vernacchio et al. 2006, Gurgel et al. 2008b). Interestingly, all-cause gastroenteritis in young children and rotavirus episodes in children of all ages were less severe in children who had recently received the rotavirus vaccine (i.e., children $<12$ months old), while older children who had received the vaccine 18 or more months earlier (i.e., more than 24 months old) had more severe episodes than the unvaccinated controls. The low incidence in young, vaccinated children may reflect some degree of protection, reducing the severity of breakthrough infections; while the increased severity in older children may result from waning vaccine immunity, as has recently been reported from Recife, Pernambuco (Correia et al. 2010). The beneficial effects of the vaccine in young children have also been observed in Australia and Mexico (Macartney et al. 2011, Quintanar-Solares et al. 2011) and our data suggest that these benefits may extend to older children. When interpreting our data, however, it is important to consider that vaccinated children were younger than unvaccinated children. Although the stratified analysis suggests that the vaccine had better protective effect in younger children, this might still be due to the effect of the higher proportion of young children in the vaccinated group that was unaccounted for by the stratified analysis (Quintanar-Solares et al. 2011).

Given the low overall rotavirus incidence rate, nonvaccinated children may have been protected from rotavirus disease (herd protection), as this effect is well described in other oral vaccines administered for other diseases, such as oral polio (Conyn-Van Spaendonck et al. 2001, Ebrahim 2001). This effect may account for the pattern of rotavirus disease observed following national immunization in the United States of America, where children too old to have received the rotavirus vaccine were found to have a reduced incidence of rotavirus disease (Tate et al. 2009). Anderson (2008) recently reported that rotavirus vaccines can be transmitted from recently vaccinated to unvaccinated children, although it is not established whether these secondary immunizations would have resulted in immunological protection, as a decreased circulation of the virus in the environment could alternatively explain this effect.

As expected, diarrhoea incidence was higher in younger children (Raúl Velázquez et al. 1993, Fernandes et al. 2000, Carneiro et al. 2005, Fischer et al. 2007, Gi- aquinto \& van Damme 2010). Similar results have been reported by Raúl Velázquez et al. (1993), who observed an incidence of 3.2 episodes/child/year in a cohort of Mexican children followed from birth, with the highest incidence occurring between seven-nive months of age, and by O'Ryan et al. (2009), who reported incidences of 0.26 and 0.57 episodes per child in the first and second six months of life, respectively, in Chile.

Similar to hospital-based reports from this region (Gurgel et al. 2007, 2009), P [4]G2 was the only genotype identified. It remains unclear whether the continued predominance of this strain can be attributed to vaccine-induced selective immune pressure or to the well-understood natural fluctuation in rotavirus genotypes (Gurgel et al. 2008a).

Leite et al. (2008) reviewed eight recent studies that reported that the predominance of $\mathrm{P}[4] \mathrm{G} 2$ and $\mathrm{P}[\mathrm{NT}] \mathrm{G} 2$ is not homogeneous across the country. Furthermore, the high prevalence of G2 reported from countries in this region (Esteban et al. 2010), where rotavirus vaccine has not been introduced, and the reported $77 \%$ efficacy of Rotarix $^{\circledR}$ against G2 in Northeast Brazil indicates that natural fluctuation of the viral genotypes may be responsible for the G2 type predominance, but a longer period of surveillance will be necessary to fully understand this phenomenon (Correia et al. 2010).

In conclusion, this study suggests that the proportion of acute diarrhoea cases caused by rotavirus were low and that the incidence of rotavirus diarrhoea decreased over the study period in both vaccinated and unvaccinated children. The all-cause diarrhoea episodes and the few rotavirus episodes observed were less severe in vaccinated young children. Rotavirus P[4]G2 was the only type of rotavirus identified. The vaccine may play a key role in decreasing rotavirus-borne diarrhoea in the community and its introduction in the immunization program is a welcome development.

\section{REFERENCES}

Anderson EJ 2008. Rotavirus vaccines: viral shedding and risk of transmission. Lancet Infect Dis 8: 642-649.

Banerjee I, Ramani S, Primrose B, Moses P, Iturriza-Gomara M, Gray JJ, Jaffar S, Monica B, Muliyil JP, Brown DW, Estes MK, Kang G 2006. Comparative study of the epidemiology of rotavirus in children from a community-based birth cohort and a hospital in south India. J Clin Microbiol 44: 2468-2474.

Bishop R 2009. Discovery of rotavirus: implications for child health. $J$ Gastroenterol Hepatol 24 (Suppl. 3): S81-85.

Carneiro NB, Diniz-Santos DR, Fagundes SQ, Neves LL, Reges RM, Lima EK, Quadros VH, Soares LE, Silva FS, Schneiter H de G, Figueiredo I, Silva LR 2005. Clinical and epidemiological aspects of children hospitalized with severe rotavirus-associated gastroenteritis in Salvador, BA, Brazil. Braz J Infect Dis 9: 525-528.

Centenari C, Gurgel RQ, Bohland AK, Oliveira DM, Faragher B, Cuevas LE 2010. Rotavirus vaccination in Northeast Brazil: a laudable intervention, but can it lead to cost-savings? Vaccine 28: 4162-4168.

Conyn-Van Spaendonck MA, de Melker HE, Abbink F, ElzingaGholizadea N, Kimman TG, van Loon T 2001. Immunity to poliomyelitis in The Netherlands. Am J Epidemiol 153: 207-214.

Correia JB, Patel MM, Nakagomi O, Montenegro FM, Germano EM, Correia NB, Cuevas LE, Parashar UD, Cunliffe NA, Nakagomi T 2010. Effectiveness of monovalent rotavirus vaccine (Rotarix) against severe diarrhea caused by serotypically unrelated G2P[4] strains in Brazil. J Infect Dis 201: 363-369. 
Ebrahim GJ 2001. Polio eradication and after. J Trop Pediatr 47: 128-130.

Esteban LE, Rota RP, Gentsch JR, Jiang B, Esona M, Glass RI, Glikmann G, Castello AA 2010. Molecular epidemiology of group A rotavirus in Buenos Aires, Argentina 2004-2007: reemergence of G2P[4] and emergence of G9P[8] strains. J Med Virol 82: 1083-1093.

Fernandes JV, Fonseca SM, Azevedo JC, Maranhão H de S, Fonseca MH, Dantas MT, Meissner R de V 2000. Rotavirus detection in feces of children with acute diarrhea. J Pediatr (Rio J) 76: 300-304.

Fischer TK, Viboud C, Parashar U, Malek M, Steiner C, Glass R, Simonsen L 2007. Hospitalizations and deaths from diarrhea and rotavirus among children $<5$ years of age in the United States, 1993-2003. J Infect Dis 195: 1117-1125.

Gentsch JR, Glass RI, Woods P, Gouvea V, Gorziglia M, Flores J, Das BK, Bhan MK 1992. Identification of group A rotavirus gene 4 types by polymerase chain reaction. J Clin Microbiol 30: 1365-1373.

Giaquinto C, van Damme P 2010. Age distribution of paediatric rotavirus gastroenteritis cases in Europe: the REVEAL study. Scand J Infect Dis 42: 142-147.

Gouvea V, Glass RI, Woods P, Taniguchi K, Clark HF, Forrester B, Fang ZY 1990. Polymerase chain reaction amplification and typing of rotavirus nucleic acid from stool specimens. J Clin Microbiol 28: 276-282.

Gurgel RG, Bohland AK, Vieira SC, Oliveira DM, Fontes PB, Barros VF, Ramos MF, Dove W, Nakagomi T, Nakagomi O, Correia JB, Cunliffe N, Cuevas LE 2009. Incidence of rotavirus and all-cause diarrhea in Northeast Brazil following the introduction of a national vaccination program. Gastroenterology 137: 1970-1975.

Gurgel RQ, Correia JB, Cuevas LE 2008a. Effect of rotavirus vaccination on circulating virus strains. Lancet 371: 301-302.

Gurgel RQ, Cuevas LE, Vieira SC, Barros VC, Fontes PB, Salustino EF, Nakagomi O, Nakagomi T, Dove W, Cunliffe N, Hart CA 2007. Predominance of rotavirus $\mathrm{P}[4] \mathrm{G} 2$ in a vaccinated population, Brazil. Emerg Infect Dis 13: 1571-1573.

Gurgel RQ, Cunliffe NA, Nakagomi O, Cuevas LE 2008b. Rotavirus genotypes circulating in Brazil before national rotavirus vaccination: a review. J Clin Virol 43: 1-8.

Leite JP, Carvalho-Costa FA, Linhares AC 2008. Group A rotavirus genotypes and the ongoing Brazilian experience - A review. Mem Inst Oswaldo Cruz 103: 745-753.

Macartney KK, Porwal M, Dalton D, Cripps T, Maldigri T, Isaacs D, Kesson A 2011. Decline in rotavirus hospitalisations following introduction of Australia's national rotavirus immunisation programme. J Paediatr Child Health doi: 10.1111/j.1440-1754.2010.01953.x.

Nakagomi T, Cuevas LE, Gurgel RG, Elrokhsi SH, Belkhir YA, Abugalia M, Dove W, Montenegro FM, Correia JB, Nakagomi O, Cunliffe NA, Hart CA 2008. Apparent extinction of non-G2 rotavirus strains from circulation in Recife, Brazil, after the introduction of rotavirus vaccine. Arch Virol 153: 591-593.

O'Ryan ML, Lucero Y, Prado V, Santolaya ME, Rabello M, Solis Y, Berrios D, O'Ryan-Soriano MA, Cortes H, Mamani N 2009. Symptomatic and asymptomatic rotavirus and norovirus infections during infancy in a Chilean birth cohort. Pediatr Infect Dis $J$ 28: 879-884.

Parashar UD, Gibson CJ, Bresse JS, Glass RI 2006. Rotavirus and severe childhood diarrhea. Emerg Infect Dis 12: 304-306.

Patel MM, Tate JE, Selvarangan R, Daskalaki I, Jackson MA, Curns AT, Coffin S, Watson B, Hodinka R, Glass RI, Parashar UD 2007. Routine laboratory testing data for surveillance of rotavirus hospitalizations to evaluate the impact of vaccination. Pediatr Infect Dis J 26: 914-919.

Quintanar-Solares M, Yen C, Richardson V, Esparza-Aguilar M, Parashar UD, Patel MM 2011. Impact of rotavirus vaccination on diarrhea-related hospitalizations among children $<5$ years of age in Mexico. Pediatr Infect Dis J 30 (Suppl. 1): S11-15.

Raúl Velázquez F, Calva JJ, Lourdes Guerrero M, Mass D, Glass RI, Pickering LK, Ruiz-Palacios GM 1993. Cohort study of rotavirus serotype patterns in symptomatic and asymptomatic infections in Mexican children. Pediatr Infect Dis J 12: 54-61.

Ruuska T, Vesikari T 1990. Rotavirus disease in Finnish children: use of numerical scores for clinical severity of diarrhoeal episodes. Scand J Infect Dis 22: 259-267.

Sáfadi MA, Berezin EN, Munford V, Almeida FJ, de Moraes JC, Pinheiro CF, Racz ML 2010. Hospital-based surveillance to evaluate the impact of rotavirus vaccination in São Paulo, Brazil. Pediatr Infect Dis J 29: 1019-1022.

Sergio JV, Leon AC 2009. Analysis of mortality from diarrheic diseases in under-five children in Brazilian cities with more than 150,000 inhabitants. Cad Saude Publica 25: 1093-1102.

Soares-Weiser K, Maclehose H, Ben-Aharon I, Goldberg E, Pitan F, Cunliffe N 2010. Vaccines for preventing rotavirus diarrhoea: vaccines in use. Cochrane Database Syst Rev 5: CD008521.

Tate JE, Panozzo CA, Payne DC, Patel MM, Cortese MM, Fowlkes AL, Parashar UD 2009. Decline and change in seasonality of US rotavirus activity after the introduction of rotavirus vaccine. Pediatrics 124: 465-471.

Valencia-Mendoza A, Bertozzi SM, Gutierrez JP, Itzler R 2008. Costeffectiveness of introducing a rotavirus vaccine in developing countries: the case of Mexico. BMC Infect Dis 8: 103.

Vernacchio L, Vezina RM, Mitchell AA, Lesko SM, Plaut AG, Acheson DW 2006. Diarrhea in American infants and young children in the community setting: incidence, clinical presentation and microbiology. Pediatr Infect Dis J 25: 2-7.

WHO - World Health Organization 2007. Rotavirus vaccines. Wkly Epidemiol Rep 82: 285-295. 